\title{
Effect of Si and Er Co-doping on Green Electroluminescence from GaN:Er ELDs
}

\author{
Rui Wang, and Andrew J Steckl
}

Department of Electrical \& Computer Engineering, University of Cincinnati, Cincinnati, OH, 45221-0030

\begin{abstract}
(Er, Si) co-doped GaN thin films were grown on Si substrates by molecular beam epitaxy (MBE) technique. Electroluminescent devices (ELDs) were fabricated and the effect of Si codoping on the performance of GaN devices was studied. Previous results with GaN:Er ELDs reported that electroluminescence (EL) was much stronger in reverse bias than in forward bias condition, indicating that the dominant factor in EL intensity was the electric field. The results reported here show the first time GaN:Er ELDs where forward bias EL is very much larger, indicating that the dominant factor is forward bias current. The electrical properties of ( $\mathrm{Si}, \mathrm{Er}$ ) co-doped $\mathrm{GaN}$ thin films are believed to be responsible for the current control mechanism.
\end{abstract}

\section{INTRODUCTION}

Rare earths are of interest in optoelectronics and photonics because of their sharp emission lines. For example, Er has emission lines at both visible (538nm and 559nm) and infrared (1.54um) wavelengths, which are very useful for display devices and optical communications. Although the emission spectrum is independent of host materials, the intensity and efficiency strongly depend on the host. It was found by Favennec ${ }^{[1]}$ that thermal quenching of the RE emission is inversely dependent on the energy bandgap of host materials. Therefore, III-nitride semiconductors are excellent host materials because of their direct wide bandgap. Moreover, robust thermal and mechanical properties of nitride semiconductors enable device applications under high temperature environment.

Sapphire and $\mathrm{SiC}$ are typically used as substrates for $\mathrm{GaN}$ thin film growth because their lattice constants are close to III-nitrides. However, GaN growth on Si substrates is very attractive for integration with Si microelectronics. ${ }^{[2]}$ The first report ${ }^{[3]}$ of electroluminescence (EL) from GaN:Er was for diodes actually grown on Si substrates and utilized a Schottky barrier structure. Subsequently, visible and IR EL emission from GaN:Er Schottky diodes was reported $^{[4]}$ on Si and sapphire substrates. Zavada et al ${ }^{[5]}$ reported EL emission from GaN:Er P-I-N LED on sapphire. In general, in all of these devices the EL emission was much stronger in reverse bias than in forward bias.

Here we report on Er-doped GaN ELD on Si wafer with Si co-doping. The effect of Si and Er co-doping on electrical properties and Er green electroluminescence in the GaN layer was studied.

\section{EXPERIMENT}

GaN thin films were grown with Er and Si in situ doping on p-type Si(111) substrates in a solid source Riber $32 \mathrm{MBE}$ system. A 20nm low temperature AlN buffer layer was first grown at $500^{\circ} \mathrm{C}$, followed by $1 \mathrm{hr}$ growth of $(\mathrm{Si}, \mathrm{Er})$ co-doped $\mathrm{GaN}$ layer. A very thin GaN capping layer 
was grown last. The substrate temperature was kept at $600^{\circ} \mathrm{C}$. The Ga effusion cell temperature was kept at $875^{\circ} \mathrm{C}$, while the $\mathrm{Si}$ and $\mathrm{Er}$ cell temperatures were kept at $1050^{\circ} \mathrm{C}$ and $860^{\circ} \mathrm{C}$. The resulting thickness of GaN thin films was approximately 500nm.

Room temperature photoluminescence (PL) measurements were performed with a Melles Griot 325nm He-Cd laser. A Princeton Instruments/Acton Research Corporation SP2550i spectrometer was used to acquire the emission spectra.

Ring diode structures were patterned by photolithography with a Karl Suss MJB3 mask aligner. ITO thin films were deposited with a Denton Vacuum sputtering system. After lift-off lithography, ITO electrodes were rapid thermal annealed. GaN ELDs doped with Er only were also fabricated with the same conditions for comparison.

\section{DISCUSSION}

PL spectra from GaN:(Er+Si) and GaN:Er samples are plotted in Figure 1. Both samples show green peaks of 538nm and 559nm from Er ions, corresponding to radiative transitions (Figure 1) from $\mathrm{Er}^{3+}$ ions energy levels ${ }^{2} \mathrm{H}_{11^{-}}{ }^{4} \mathrm{I}_{15 / 2}$ and ${ }^{4} \mathrm{~S}_{3 / 2}{ }^{4} \mathrm{I}_{15 / 2}$. The peak intensities are similar if we subtract yellow band background from the spectra. There is also a small peak around $369 \mathrm{~nm}$ corresponding to free exciton emission from GaN bandgap for both samples. No Sirelated peak was observed in the PL spectra of ( $\mathrm{Si}, \mathrm{Er})$ co-doped $\mathrm{GaN}$ thin films.
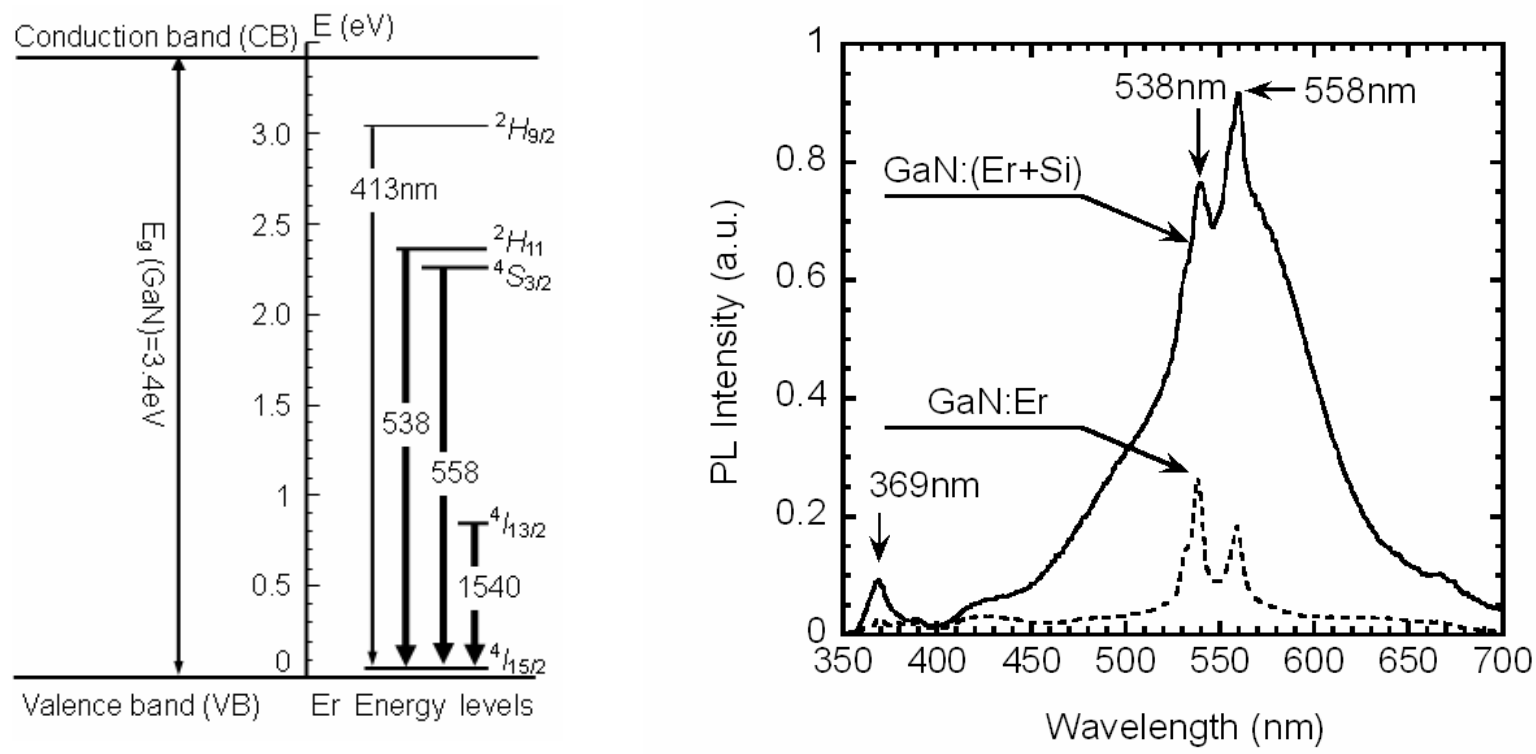

Figure 1. Energy levels of $\mathrm{Er}^{3+}$ ions and $\mathrm{GaN}$ valence and conduction bands (left); photoluminescence spectra from GaN doped with Er only, and co-doped with Er and Si (right).

Ring diode structures were fabricated with both ( $\mathrm{Si}, \mathrm{Er}$ ) co-doped $\mathrm{GaN}$ samples and Er only GaN samples. The device structure is shown in Figure 2. ITO electrodes were patterned as inner and outer rings. Ag paste was used to connect the outer ring to the Si substrate. Currentvoltage measurements were performed for all GaN ELDs with Labview controlled HP 6634B DC power supply. Typical results are plotted in Figure 3. Er doped GaN ELDs have similar I-V 
under forward and reverse bias, while ( $\mathrm{Si}, \mathrm{Er}$ ) co-doped GaN ELDs exhibit strong I-V rectification characteristics.

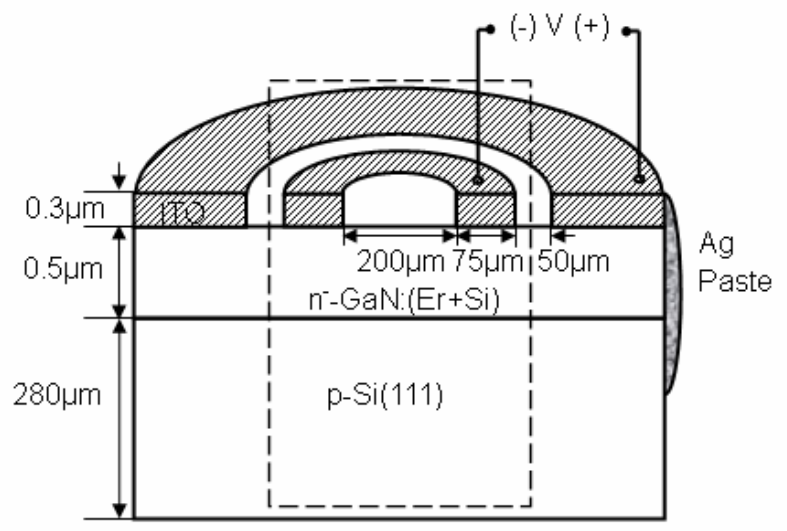

Figure 2. Diagram of (Er, Si) co-doped GaN ELDs.

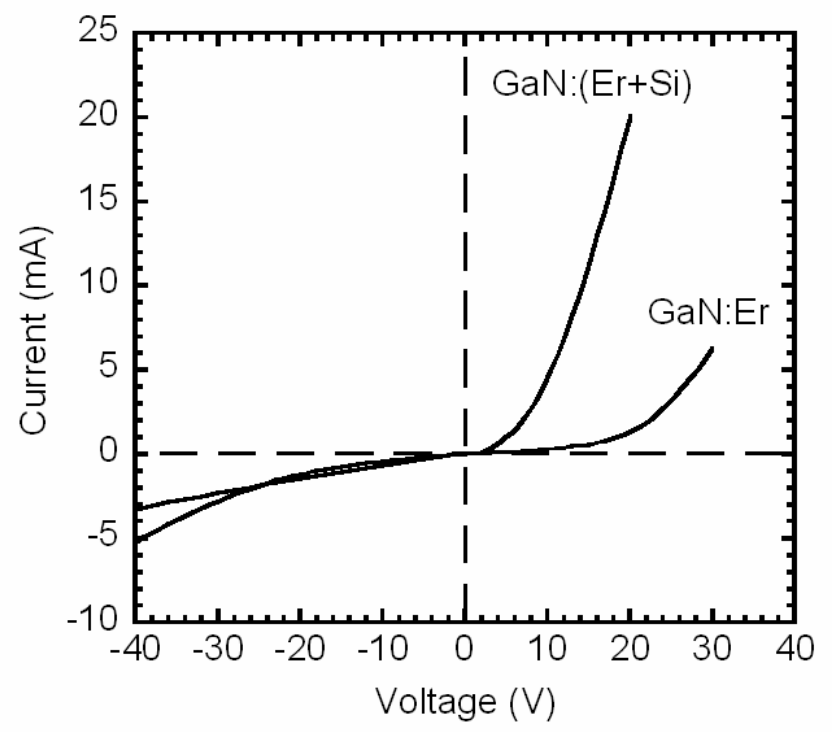

Figure 3. I-V characteristics of (Er, Si) co-doped GaN and Er doped GaN ELDs.

Electroluminescence measurements were performed on ( $\mathrm{Si}, \mathrm{Er}$ ) co-doped GaN ELDs. Visible (green) EL spectra are shown in Figure 4 at several bias levels. The EL emission is clearly very strong under forward bias, with the intensity proportional to the current. Under reverse bias as high as $100 \mathrm{~V}$, little or no emission was observed. Compared to previous results on RE doped GaN ELDs, this is first time that GaN:RE ELDs were observed to have strong emission under forward bias with no emission under reverse bias. The luminescence dependence on voltage and current was also obtained and the results are shown in Figure 5. 


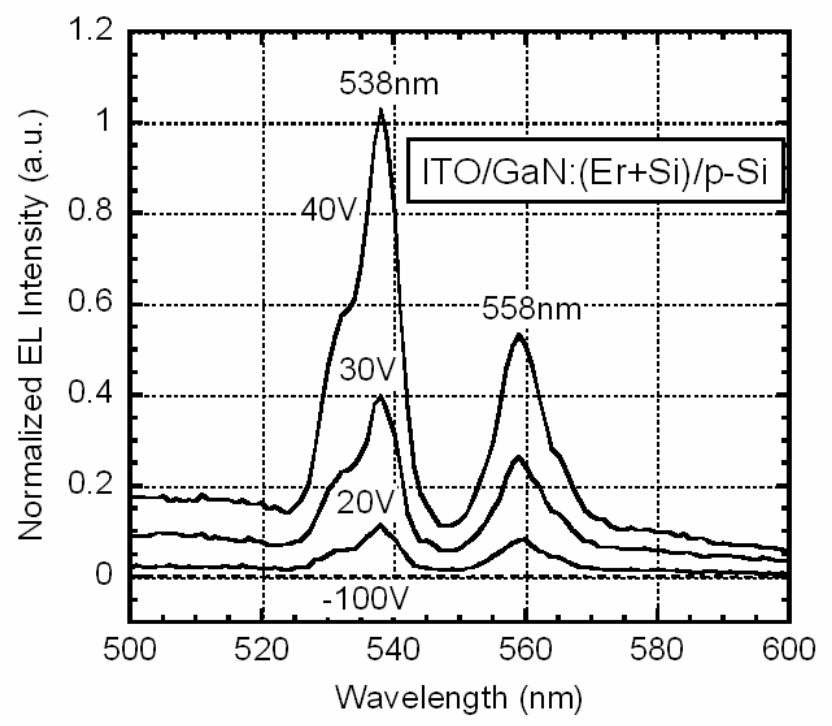

Figure 4. Visible EL spectra from ITO/GaN:(Si+Er)/p-Si(111) devices at several bias levels.
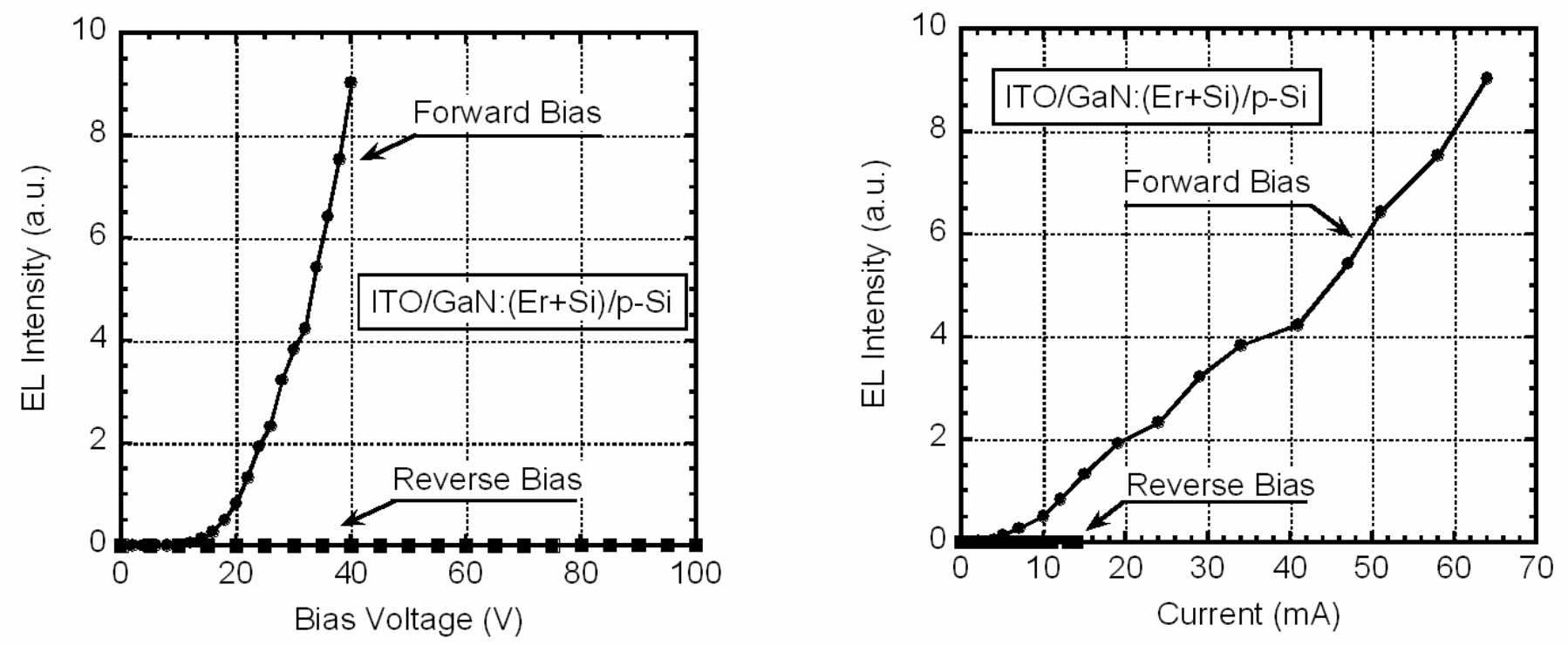

Figure 5. EL intensity dependence on voltage and current conditions from ITO/GaN:(Si+Er)/p-Si(111) device under forward and reverse bias.

Undoped GaN thin films grown in our lab typically show weak n-type conductivity with electron concentration around $10^{17} \mathrm{~cm}^{-3}$. It is believed ${ }^{[6]}$ that the nitrogen vacancy $\left(\mathrm{N}_{\mathrm{v}}\right)$ acts as the donor and accounts for such n-type conductivity. However, RE-doped GaN is generally highly insulating. The change to an insulating GaN:RE layer is not fully understood, but it is generally thought that RE ions act as electron trap centers in the GaN bandgap. 
With Si co-doping in GaN:RE thin films, $\mathrm{Si}$ atoms act as donors and provide a significant source of electrons. The electrical properties of GaN:Er thin films have been modified from highly resistive to weakly n-type. The sheet resistance of $(\mathrm{Si}, \mathrm{Er})$ co-doped $\mathrm{GaN}$ thin films was measured as $1.15 \times 10^{5} \Omega / \square$ by a six point probe meter, whereas the Er-doped-only GaN has a resistance too large to be measured $\left(>10^{10} \Omega / \square\right)$. Combined with the p-type $\operatorname{Si}(111)$ substrate, a $\mathrm{pn}^{-}$heterojunction is formed at the interface between the GaN layer and the Si layer, shown as the dashed rectangle area in Figure 2. The electrical properties of the pn heterostructure strongly affect the I-V characteristics of GaN:Er ELDs under different DC bias.

Generally, there are two main mechanisms for RE luminescence: electron-hole recombination and hot carriers impact excitation. ${ }^{[7]}$ For EL emission, as electrons and holes are injected into the active GaN layer under forward bias, they recombine and transfer their energy to neighboring RE ions. Some of the excited RE ions relax radiatively, resulting in EL emission. Under reverse bias, hot electrons injected into the GaN layers collide with RE ions and transfer their energy to RE ions directly. For RE-doped-only GaN ELDs, the insulating GaN:RE layer acts a high barrier layer and limits the current which can go through the layer. Since the Si codoped GaN:RE layer is much more conducting, the $\mathrm{pn}^{-}$heterojunction experiences enhanced current flow under forward bias, but acts as a barrier for electrons under reverse bias. Although a few hot electrons can tunnel through the $\mathrm{GaN}$ :( $\mathrm{Si}+\mathrm{Er})$ layer, their energy probably is not sufficient for impact excitation mechanism, resulting in little or no emission.

\section{CONCLUSIONS}

In conclusion, we have reported GaN thin films co-doped with Er and Si grown by MBE. And we have fabricated GaN ELDs on Si substrates. The effect of Si and Er co-doping on the electrical and luminescent properties of ( $\mathrm{Si}, \mathrm{Er}$ ) co-doped GaN ELDs was studied. The co-doped $(\mathrm{Er}+\mathrm{Si}) \mathrm{GaN}$ ELDs have achieved for the first time current controlled luminescence.

\section{ACKNOWLEDGMENTS}

This work is supported by an ARO grant. The authors would like to acknowledge Dr. John Zavada for his kind support and encouragement.

\section{REFERENCES}

[1] P. N. Favennec, H. L'Haridon, M. Salvi, D. Moutonnet, and Y. Le Guillou, "Luminescence of erbium implanted in various semiconductors: IV, III-V and II-VI materials," Elec. Lett., vol. 25, p. 718, 1989.

[2] A. J. Steckl, J. H. Park, and J. M. Zavada, "Prospects for rare earth doped GaN lasers on Si," Materials Today, vol. 10, pp. 20-27, 2007.

[3] A. J. Steckl, M. Garter, R. Birkhahn, and J. Scofield, "Green electroluminescence from Er-doped GaN Schottky barrier diodes," Appl. Phys. Lett., vol. 73, pp. 2450-2452, 1998.

[4] M. J. Garter and A. J. Steckl, "Temperature behavior of visible and infrared electroluminescent devices fabricated on erbium-doped GaN," IEEE Trans. on electron devices, vol. 49, pp. 48-54, 2002. 
[5] J. M. Zavada, S. X. Jin, N. Nepal, J. Y. Lin, H. X. Jiang, P. Chow, and B. Hertog, "Electroluminescent properties of erbium-doped III-V light-emitting diodes," Appl. Phys. Lett., vol. 84, pp. 1061-1063, 2004.

[6] D. W. Jenkins and J. D. Dow, "Electronic structures and doping of $\operatorname{InN}, \operatorname{In}_{\mathrm{x}} \mathrm{Ga}_{1-\mathrm{x}} \mathrm{N}$, and $\mathrm{In}_{\mathrm{x}} \mathrm{Al}_{1-\mathrm{x}} \mathrm{N}, "$ Phys. Rev. B, vol. 39, pp. 3317-3329, 1989.

[7] A. J. Steckl, J. Heikenfeld, M. Gartner, R. Birkhahn, and D. S. Lee, "Rare Earth Doped Gallium Nitride - Light Emission from Ultraviolet to Infrared," Compound Semiconductor, vol. 6, pp. 48-52, 2000. 